\title{
STABILITY OF NONLINEAR URYSOHN INTEGRAL EQUATIONS VIA GLOBAL DIFFEOMORPHISMS AND IMPLICIT FUNCTION THEOREMS
}

\author{
DOROTA BORS
}

Communicated by Jurgen Appell

\begin{abstract}
In the paper, we prove the existence, uniqueness and differentiable dependence of solutions for some nonlinear Urysohn integral equations on parameters. Some sufficient conditions for the nonlinear integral operator of the Urysohn type to be a diffeomorphism are stated. Global invertibility of the Urysohn operator in a certain Sobolev space is ascertained. Consequently, global solvability of Urysohn equations is claimed. Similar results are obtained for some nonlinear Urysohn integral equations with controls by the use of the global implicit function theorem published in the recent paper by Idczak. The proofs of global diffeomorphisms and global implicit functions theorems, the main tools used in the paper, rely in an essential way on the mountain pass theorem. Applications of results to some specific nonlinear Urysohn integral equations are also presented.
\end{abstract}

1. Introduction. We consider the following nonlinear Urysohn integral equations of the form

$$
\begin{gathered}
\lambda x(t)+\int_{\alpha}^{\beta} f(t, \tau, x(\tau)) d \tau=z(t) \\
\lambda x(t)+\int_{\alpha}^{\beta} g(t, \tau, x(\tau), u(\tau)) d \tau=z(t),
\end{gathered}
$$

2010 AMS Mathematics subject classification. Primary 45G15, 45Q05, 47B38, $47 \mathrm{H} 30$.

Keywords and phrases. Urysohn integral equation, global diffeomorphism theorem, implicit function theorem, mountain pass theorem, Palais-Smale condition, control problems, continuous dependence and differentiable dependence on data.

Received by the editors on November 26, 2014, and in revised form on April 8, 2015. 
where $t \in[\alpha, \beta], \lambda \in \mathbb{R}, f: P \times \mathbb{R}^{n} \rightarrow \mathbb{R}^{n}, g: P \times \mathbb{R}^{n} \times \mathbb{R}^{m} \rightarrow \mathbb{R}^{n}, n \geq 1$, $m \geq 1, P=[\alpha, \beta] \times[\alpha, \beta], x, z \in \widetilde{H}_{0}^{1}$ and $u \in L^{2}:=L^{2}\left([\alpha, \beta], \mathbb{R}^{m}\right)$. By $\widetilde{H}_{0}^{1}$, we shall denote the space $\widetilde{H}_{0}^{1}\left([\alpha, \beta], \mathbb{R}^{n}\right)$ of absolutely continuous functions defined on $[\alpha, \beta]$ with a square integrable derivative satisfying the condition $x(\alpha)=0$ with the norm $\|x\|_{\tilde{H}_{0}^{1}}^{2}=\int_{\alpha}^{\beta}\left|x^{\prime}(t)\right|^{2} d t$.

We obtain the existence of a unique solution to the nonlinear Urysohn system of equations (1.1) in the space $\widetilde{H}_{0}^{1}$ that depends continuously and even differentiably on the right hand side from $\widetilde{H}_{0}^{1}$, provided $f$ grows linearly with respect to $x$ and the growth is controlled by parameter $|\lambda|$. The results rely on the global diffeomorphisms theorem from the paper [13] by Idczak, et al., combining a variational approach with the topological one. Similar results can be obtained by the version of global implicit function theorem published recently in [12] applied to (1.2) the nonlinear Urysohn equations with control $u$.

Integral equations can be derived from models that appear in various fields of science, for references, see for example, books by Corduneanu [6] or by Gripenberg, et al., [11]. Several kinds of integral operators considered therein include those of Fredholm, Volterra, Hammerstein, Urysohn and the Wiener-Hopf type. For references on Hammerstein equations, being the special case of Urysohn equation, see, for example, among others, $[\mathbf{4}, \mathbf{5}, \mathbf{7}, \mathbf{8}, \mathbf{9}, \mathbf{1 0}, \mathbf{2 1}]$. Interest in the Hammerstein equation stems mainly from the fact that several problems that arise in differential equations, for instance, elliptic boundary value problems, whose linear parts possess the Green function, can, as a rule, be transformed into an equation involving Hammerstein integral operator. Among these, we mention the paper [17] or the BVPs on real line of Hammerstein and Wiener-Hopf type, see, e.g., [2, 21].

In the paper, we consider the nonlinear integral operators of Urysohn type, with the nonautonomous kernel, i.e., depending not only on the unknown function variable but also on time and the integrable variable, some parameters and even on the right hand side. Integral operators of Urysohn type which are examined in the paper have the following forms

$$
F(x)(t)=\lambda x(t)+\int_{\alpha}^{\beta} f(t, \tau, x(\tau)) d \tau
$$




$$
G(x, u, z)(t)=\lambda x(t)+\int_{\alpha}^{\beta} g(t, \tau, x(\tau), u(\tau)) d \tau-z(t),
$$

where $\lambda \in \mathbb{R}, t \in[\alpha, \beta]$ and $x, z \in \widetilde{H}_{0}^{1}, u \in L^{2}$.

Without going into details, under some suitable assumptions imposed on the function $f$, it is possible to prove that the operator $F: \widetilde{H}_{0}^{1} \rightarrow \widetilde{H}_{0}^{1}$ is a diffeomorphism, that is, $F\left(\widetilde{H}_{0}^{1}\right)=\widetilde{H}_{0}^{1}$ and that there exists an inverse operator $F^{-1}$ while both $F, F^{-1}$ are Fréchet differentiable at every point from $\widetilde{H}_{0}^{1}$. In other words, $F$ is Fréchet differentiable at every point $x \in \widetilde{H}_{0}^{1}$ and, for every $z \in \widetilde{H}_{0}^{1}$, there exists a unique solution $x_{z} \in \widetilde{H}_{0}^{1}$ to the operator equation $F(x)=z$ depending continuously on $z$ and the operator $\widetilde{H}_{0}^{1} \ni z \rightarrow x_{z} \in \widetilde{H}_{0}^{1}$ is continuously Fréchet differentiable. To obtain the announced results we apply the theorem on global diffeomorphisms in a Hilbert space, published in the paper by Idczak, et al., see [13, Theorem 3.1].

As far as solvability and differentiable dependence on data $(u, z)$ of (1.2) are concerned, analogous results to those obtained for (1.1) can be stated. In this case, instead of the global diffeomorphisms theorem applied to $F$ given by (1.3), the global implicit function theorem involving the operator $G$ given by (1.4) is employed. In the paper, the version of global implicit function theorem published in the recent paper by Idczak, see [12, Theorem 4.1] is recalled. In this case, assumptions imposed on $g$ with respect to $t, \tau, x$ variables are analogous to those required for $f$ with some extra regularity and growth conditions with respect to the variable $u$.

The rest of this paper is structured as follows. In Section 2, we present theorems on the global diffeomorphisms and on the global implicit function employed in the paper. Section 3 contains some regularity, growth and quantitative assumptions as well as some auxiliary lemmas. In Section 4, we focus our attention on some sufficient condition for the derivative of $F$ to be bijective whereas in Section 5 some sufficient condition for the functional involving the Urysohn operator to satisfy the Palais-Smale condition is addressed. The main results on solvability and differentiable dependence on data are gathered in Section 6. In this section, the examples of some nonlinear Urysohn integral operators illustrating the presented results are included. Section 7 is devoted to the nonlinear Urysohn integral equations with controls. Fi- 
nally, in the last section concluding remarks are stated.

2. Global diffeomorphisms and global implicit function theorems. We begin with the theorem that provides sufficient conditions for some general operator to be a diffeomorphism, see [13, Theorem 3.1], namely, we have the following theorem on a global diffeomorphism.

Theorem 2.1. Let $H$ be a real Hilbert space. If $\mathcal{F}: H \rightarrow H$ is a $C^{1}$-mapping such that

(a) for any $x \in H$ the derivative $\mathcal{F}^{\prime}(x): H \rightarrow H$ is bijective,

(b) for any $y \in H$ the functional

$$
\Psi_{y}: H \ni x \longmapsto \frac{1}{2}\|\mathcal{F} x-y\|_{H}^{2} \in \mathbb{R}
$$

satisfies the Palais-Smale condition,

then $\mathcal{F}$ is a diffeomorphism.

Let $\psi: H \rightarrow \mathbb{R}$ be of $C^{1}$ class. A sequence $\left\{x_{k}\right\}_{k \in \mathbb{N}}$ is referred to as a Palais-Smale sequence for functional $\psi$ if, for some $M>0$, any $k \in \mathbb{N},\left|\psi\left(x_{k}\right)\right| \leq M$ and $\psi^{\prime}\left(x_{k}\right) \rightarrow 0$ as $k \rightarrow \infty$. Moreover, we say that the functional $\psi$ satisfies the Palais-Smale condition if any Palais-Smale sequence admits a convergent subsequence.

Furthermore, by the assumption $(a)$ and the bounded inverse theorem, we infer that, for any $x \in H$, there is a constant $\alpha_{x}>0$ satisfying, for any $h \in H$,

$$
\left\|\mathcal{F}^{\prime}(x) h\right\|_{H} \geq \alpha_{x}\|h\|_{H} .
$$

Therefore, Theorem 2.1 is equivalent to [13, Theorem 3.1].

In the proof of Theorem 2.1 the renowned variational mountain pass theorem is applied. For more details, we refer the reader to the vast literature on the subject, for example, see $[\mathbf{1 , 1 4}, \mathbf{1 8}]$. In the statement of the mountain pass theorem the following notation is used. Let $e \neq 0$ be a point of $H$ and $W_{e}$ the set of the form

$$
W_{e}=\{U \subset H: U \text { is open, } 0 \in U \text { and } e \notin \bar{U}\},
$$


where $B_{\rho}$ denotes the following ball:

$$
B_{\rho}=\left\{x \in H:\|x\|_{H}<\rho\right\} .
$$

Theorem 2.2 (Mountain pass theorem). Let $H$ be a real Banach space, $\psi: H \rightarrow \mathbb{R}$ a $C^{1}$-mapping satisfying the Palais-Smale condition and $\psi(0)=0$. If

(i) there are some constants $\rho>0$ and $\alpha>0$ such that $\left.\psi\right|_{\partial B_{\rho}} \geq \alpha$,

(ii) there is a point $e \in H \backslash \bar{B}_{\rho}$ such that $\psi(e) \leq 0$,

then $c=\sup _{U \in W_{e}} \inf _{x \in \partial U} \psi(x)$ is the critical value of $\psi$ and $c \geq \alpha$.

Sketch of the proof of Theorem 2.1. We sketch, for the sake of completeness, the proof, following the lines of the proof of $[\mathbf{1 3}$, Theorem 3.1]. Fix $y \in H$. Since the functional $\Psi_{y} \in C^{1}$ is bounded from below and satisfies the Palais-Smale condition, by [22, Corollary 2.5], it attains a minimum $x^{*}$, and thus its derivative at $x^{*}$ is zero, i.e., for any $h \in H$

$$
0=\Psi_{y}^{\prime}\left(x^{*}\right) h=\left\langle\mathcal{F}\left(x^{*}\right)-y, \mathcal{F}^{\prime}\left(x^{*}\right) h\right\rangle .
$$

Consequently, since $\operatorname{Im} \mathcal{F}^{\prime}\left(x^{*}\right)=H$, one obtains $\mathcal{F}\left(x^{*}\right)=y$. Thus, we have proved that $\mathcal{F}$ has to be surjective. Now suppose, on the contrary, that $\mathcal{F}$ is not injective, so there exists $x_{1} \neq x_{2}$ such that $\mathcal{F}\left(x_{1}\right)=\mathcal{F}\left(x_{2}\right)$. Define

$$
\psi(x)=\frac{1}{2}\left\|\mathcal{F}\left(x+x_{1}\right)-\mathcal{F}\left(x_{2}\right)\right\|_{H}^{2} .
$$

Observe that $\psi$ is a $C^{1}$-mapping that enjoys the Palais-Smale property and satisfies the assumptions of the mountain pass theorem with $e=x_{2}-x_{1}$ and $\alpha=(1 / 8) \alpha_{x_{1}}^{2} \rho^{2}$, where $\rho<\left\|x_{2}-x_{1}\right\|_{H}$. Indeed, a simple calculation shows

$$
\psi(e)=\psi\left(x_{2}-x_{1}\right)=0=\psi(0) .
$$

Moreover, for sufficiently small $\|x\|_{H}=\rho$, by a direct application of (2.1) at the point $x_{1}$ and the Taylor formula, one gets

$$
\begin{aligned}
\psi(x) & =\frac{1}{2}\left\|\mathcal{F}\left(x+x_{1}\right)-\mathcal{F}\left(x_{1}\right)\right\|_{H}^{2} \\
& \geq \frac{1}{2}\left(\left\|\mathcal{F}^{\prime}\left(x_{1}\right) x\right\|_{H}-\frac{1}{2} \alpha_{x_{1}}\|x\|_{H}\right)^{2}
\end{aligned}
$$




$$
\geq \frac{1}{2}\left(\left(1-\frac{1}{2}\right) \alpha_{x_{1}}\|x\|_{H}\right)^{2} .
$$

Consequently, there exists a critical point $x^{*} \in H$ for $\psi$ such that $\psi\left(x^{*}\right)>0=\psi(0)$ and, for any $h \in H$, the equality $0=\psi^{\prime}\left(x^{*}\right) h=$ $\left\langle\mathcal{F}\left(x^{*}+x_{1}\right)-\mathcal{F}\left(x_{2}\right), \mathcal{F}^{\prime}\left(x^{*}+x_{1}\right) h\right\rangle$ holds. Therefore, due to $\operatorname{Im} \mathcal{F}^{\prime}\left(x^{*}+\right.$ $\left.x_{1}\right)=H$, we get $\mathcal{F}\left(x^{*}+x_{1}\right)-\mathcal{F}\left(x_{2}\right)=0$, which contradicts $\psi\left(x^{*}\right)>$ 0 .

We end this section by stating, without proof, a version of the theorem on a global implicit function published in [12], see Theorem 4.1. The proof of this theorem is analogous to the proof of Theorem 2.1 and can be found in [12].

Theorem 2.3. Let $Y$ and $H$ be two real Hilbert spaces. If $\mathcal{F}: H \times Y \rightarrow$ $H$ is of the $C^{1}$ class and

(i) differential $\mathcal{F}_{x}(x, y): H \rightarrow H$ is bijective for any $(x, y) \in H \times Y$,

(ii) the functional $\Psi_{y}(x)=(1 / 2)\|\mathcal{F}(x, y)\|_{H}^{2}$ satisfies the PalaisSmale condition for any $y \in Y$,

then there exists a unique function $\Lambda: Y \rightarrow H$ of the $C^{1}$ class such that equations $\mathcal{F}(x, y)=0$ and $\Lambda(y)=x$ are equivalent in $H \times Y$ and, for any $y \in Y$,

$$
\Lambda^{\prime}(y)=-\left[\mathcal{F}_{x}(\Lambda(y), y)\right]^{-1} \circ \mathcal{F}_{x}(\Lambda(y), y) .
$$

Remark 2.4. It should be noted that originally in Idczak's papers $[\mathbf{1 2}, \mathbf{1 3}]$ the domain of the diffeomorphism $\mathcal{F}$ is a Banach space. In a personal communication from Idczak it was pointed out that, if the parallelogram law is obeyed in the Banach space, then the isomorphism $\mathcal{F}^{\prime}(x)$ between the Banach and the Hilbert spaces implies that in fact the Banach space is a Hilbert space. On the other hand, if the parallelogram law is not true as, for example, in $\mathbb{R}^{2}$ with $|\cdot|_{p}$ norm for $p>2$, then the isomorphism does not guarantee the Banach space to be a Hilbert one. This is the case if one takes the identity operator acting in $\mathbb{R}^{2}$ with two different but equivalent norms: $|\cdot|_{2}$ and $|\cdot|_{p}$ with $p>2$. Moreover, an infinite-dimensional counterexample can also be constructed in a separable Hilbert space by introducing a new, equivalent norm not obeying the parallelogram law. However, the very existence of the isomorphism $\mathcal{F}^{\prime}(x)$ allows us to introduce the 
scalar product with the induced norm equivalent to the original one. Therefore, as was pointed out by the anonymous referees, the existence of an isomorphism thus implicitly implies the underlying Banach space to be, in fact, a Hilbert one, with an equivalent norm. In our case, apart from the above argument, also from the applicable point of view, the use of the Hilbert space is sufficient, although we do not explicitly make use of the scalar product in the domain of the operator $\mathcal{F}$.

3. Assumptions. In what follows, we shall impose on the function $f$ in (1.3) the following assumptions:

A1(a) the function $f(\cdot, \tau, \cdot)$ is continuous on the set $Q:=[\alpha, \beta] \times \mathbb{R}^{n}$ for almost every $\tau \in[\alpha, \beta]$,

A1(b) there exists $f_{t}(\cdot, \tau, \cdot)$ and it is continuous on $Q$ for almost every $\tau \in[\alpha, \beta]$

A1(c) there exists $f_{x}(\cdot, \tau, \cdot)$, and it is continuous on set $Q$ for almost every $\tau \in[\alpha, \beta]$,

A1(d) there exists $f_{t x}(\cdot, \tau, \cdot)$, and it is continuous on set $Q$ for almost every $\tau \in[\alpha, \beta]$;

A2(a) the function $f(t, \tau, x)$ is measurable with respect to $\tau$ for $(t, x) \in Q$ and locally bounded with respect to $x$ for $(t, \tau) \in P$, i.e., for every $\rho>0$ there exists $l_{\rho}>0$ such that, for $(t, \tau) \in P$ and $x \in \bar{B}_{\rho}=\left\{x \in \mathbb{R}^{n} ;|x| \leq \rho\right\}$, we have $|f(t, \tau, x)| \leq l_{\rho}$,

A2(b) the function $f_{t}(t, \tau, x)$ satisfies A2(a) with $f_{t}$ instead of $f$,

A2(c) the function $f_{x}(t, \tau, x)$ satisfies A2(a) with $f_{x}$ instead of $f$,

A2(d) the function $f_{t x}(t, \tau, x)$ satisfies A2(a) with $f_{t x}$ instead of $f$;

A3 $f(\alpha, \tau, x)=0$ and $f_{x}(\alpha, \tau, x)=0$ for any $x \in \mathbb{R}^{n}$ and almost every $\tau \in[\alpha, \beta]$

A4 $\left|f_{x t}(t, \tau, x)\right|<|\lambda| /(\beta-\alpha)$ for any $x \in \mathbb{R}^{n}$ and $(t, \tau) \in P$;

A5(a) $\left|f_{t}(t, \tau, x)\right| \leq a(t, \tau)|x|+b(t, \tau)$ where $(t, \tau) \in P, a, b \in$ $L^{2}(P, \mathbb{R})$,

A5(b) $\|a\|_{L^{2}(P, \mathbb{R})}<(\sqrt{2}|\lambda|) /(2(\beta-\alpha))$.

To prove that $F: \widetilde{H}_{0}^{1} \rightarrow \widetilde{H}_{0}^{1}$ defined by (1.3) is a diffeomorphism, we need, besides regularity $A 1, A 2$ and technical $A 3$ assumptions imposed on function $f$, some growth and quantitative assumptions which are formulated in $A 4$ and $A 5$.

One can prove in a similar manner as in $[2,3]$ the following lemmas. 
Lemma 3.1. If the function $f$ satisfies $A 1(a), A 1(b), A 2(a), A 2(b)$ and $A 3$, then the operator $F: \widetilde{H}_{0}^{1} \rightarrow \widetilde{H}_{0}^{1}$ is well defined by (1.3).

Lemma 3.2. Suppose that the function $f$ satisfies $A 1(a), A 1(c)$, $A 2(a), A 2(c)$ and $A 3$. Then the operator $F$, defined by (1.3), is continuously Fréchet differentiable at any point $x_{0} \in \widetilde{H}_{0}^{1}$ while, for any $h \in \widetilde{H}_{0}^{1}$ and $t \in[\alpha, \beta]$,

$$
F^{\prime}\left(x_{0}\right) h(t)=\lambda h(t)+\int_{\alpha}^{\beta} f_{x}\left(t, \tau, x_{0}(\tau)\right) h(\tau) d \tau .
$$

4. Bijectivity of the Urysohn operator's derivative. Let $x_{0} \in$ $\widetilde{H}_{0}^{1}$ be a fixed but arbitrary function and $T: \widetilde{H}_{0}^{1} \rightarrow \widetilde{H}_{0}^{1}$ a linear operator defined, for any $w \in \widetilde{H}_{0}^{1}$ and $t \in[\alpha, \beta]$, by

$$
(T w)(t)=\int_{\alpha}^{\beta} f_{x}\left(t, \tau, x_{0}(\tau)\right) w(\tau) d \tau
$$

where the function $f$ defines the operator $F$ given in (1.3). Then, for any $k \in \mathbb{N}, t \in[\alpha, \beta]$ and $w \in \widetilde{H}_{0}^{1}$, let us estimate the following sequence of iterations:

$$
\left(T^{k+1} w\right)(t)=\left(T\left(T^{k} w\right)\right)(t)=\int_{\alpha}^{\beta} f_{x}\left(t, \tau, x_{0}(\tau)\right)\left(T^{k} w\right)(\tau) d \tau,
$$

with

$$
\left(T^{0} w\right)(t)=w(t) .
$$

First, let $M$ be a constant such that

$$
M=\|w\|_{\infty}:=\sup _{t \in[\alpha, \beta]}|w(t)|,
$$

and, for every $\rho>0$, let $l_{\rho}$ be defined as

$$
l_{\rho}:=\sup _{\substack{(t, \tau) \in P \\|x|<\rho}}\left|f_{x}(t, \tau, x)\right| .
$$

We shall prove the following lemma. 
Lemma 4.1. If $A 1(c), A 2(c)$ and $A 3$ are satisfied, then

$$
\left|\left(T^{k} w\right)(t)\right| \leq(\beta-\alpha)^{k} l_{\rho}^{k} M, \quad \text { for } k \in \mathbb{N}_{0} \text { and } t \in[\alpha, \beta] .
$$

Proof. Similarly to [2, Lemma 8], from (4.1)-(4.3) and, by the assumptions of the lemma, we get the following estimates

$$
\begin{aligned}
& \left|\left(T^{1} w\right)(t)\right| \leq(\beta-\alpha) l_{\rho} M, \\
& \left|\left(T^{2} w\right)(t)\right| \leq \int_{\alpha}^{\beta}\left|f_{x}\left(t, \tau, x_{0}(\tau)\right)\right|\left|\left(T^{1} w\right)(\tau)\right| d \tau \leq(\beta-\alpha)^{2} l_{\rho}^{2} M, \\
& \left|\left(T^{3} w\right)(t)\right| \leq \int_{\alpha}^{\beta}\left|f_{x}\left(t, \tau, x_{0}(\tau)\right)\right|\left|\left(T^{2} w\right)(\tau)\right| d \tau \leq(\beta-\alpha)^{3} l_{\rho}^{3} M .
\end{aligned}
$$

To finish the proof, we proceed by induction to get estimate (4.4).

Next, we shall formulate and prove the existence and uniqueness results for the linear integral equation of the form

$$
\lambda h(t)+\int_{\alpha}^{\beta} f_{x}\left(t, \tau, x_{0}(\tau)\right) h(\tau) d \tau=w(t), \quad \text { for } t \in[\alpha, \beta]
$$

where $x_{0} \in \widetilde{H}_{0}^{1}$ and $w \in \widetilde{H}_{0}^{1}$ are arbitrarily fixed. Equivalently, we shall demonstrate that the linear integral operator defined by the left hand side of equation (4.5) is a bijection from $\widetilde{H}_{0}^{1}$ onto $\widetilde{H}_{0}^{1}$.

Before the proof of the announced results, for the sake of completeness, we recall a theorem on spectral radius known as the standard fact from functional analysis. If $\mathcal{T}$ denotes a bounded and continuous operator in a Banach space $X$. Then we can decompose $\mathbb{C}$ into the resolvent of $\mathcal{T}$ defined as

$$
\rho(\mathcal{T})=\{\lambda \in \mathbb{C}: \mathcal{T}-\lambda \mathcal{I} \text { is a bijection on } X\},
$$

and the spectrum of $\mathcal{T}$ defined as

$$
\sigma(\mathcal{T})=\mathbb{C} \backslash \rho(\mathcal{T})=\{\lambda \in \mathbb{C}: \mathcal{T}-\lambda \mathcal{I} \text { is a bijection on } X\} .
$$

For any bounded and continuous operator $\mathcal{T}$, we can define the spectral radius of $\mathcal{T}$ by the formula

$$
r(\mathcal{T})=\limsup _{k \rightarrow \infty}\left\|\mathcal{T}^{k}\right\|^{1 / k}
$$


which is finite due to the estimate

$$
r(\mathcal{T}) \leq\|\mathcal{T}\|
$$

Moreover, we have, following, for example [19, Theorem VI.6] and [23, Theorem VIII.2.3], the theorem on the spectral radius.

Theorem 4.2. For any $|\lambda|>r(\mathcal{T}), \lambda \in \rho(\mathcal{T})$.

Remark 4.3. The spectrum of $\mathcal{T}$ is contained in the closed ball of radius $r(\mathcal{T})$, i.e., $\sigma(\mathcal{T}) \subset \bar{B}_{r(\mathcal{T})}=\{\lambda \in \mathbb{C}:|\lambda| \leq r(\mathcal{T})\}$.

We are now in a position to state and prove the lemma on solvability of the linear integral equation (4.5). In other words, the mapping $\widetilde{H}_{0}^{1} \ni h \rightarrow F^{\prime}(x) h \in \widetilde{H}_{0}^{1}$ is bijective for any $x \in \widetilde{H}_{0}^{1}$, which is assumption (a) from Theorem 2.1.

Lemma 4.4. Let us assume that the function $f$ satisfies conditions $A 1, A 2, A 3$ and

$$
|\lambda|>l_{\rho}(\beta-\alpha)
$$

where $l_{\rho}=\sup _{(t, \tau) \in P,|x|<\rho}\left|f_{x t}(t, \tau, x)\right|$. Then, for any $x_{0} \in \widetilde{H}_{0}^{1}, \rho>0$ such that $\left\|x_{0}\right\|_{\infty} \leq \rho$ and any $w \in \widetilde{H}_{0}^{1}$, equation (4.5) possesses a unique solution in $\widetilde{H}_{0}^{1}$.

Proof. From (4.1)-(4.4), for any $k \in \mathbb{N}, t \in[\alpha, \beta]$ and $w \in \widetilde{H}_{0}^{1}$, we have

$$
\left(T^{k+1} w\right)(t)=\int_{\alpha}^{\beta} f_{x}\left(t, \tau, x_{0}(\tau)\right)\left(T^{k} w\right)(\tau) d \tau
$$

with

$$
\left(T^{0} w\right)(t)=w(t)
$$

By $A 1, A 2, A 3$ and estimate (4.4), similarly as in [2] one can deduce that $\left(T^{k} w\right) \in \widetilde{H}_{0}^{1}$ for any $w \in \widetilde{H}_{0}^{1}$ and $k \in \mathbb{N}$ and, moreover, one has

$$
\begin{aligned}
\left\|\left(T^{k} w\right)\right\|_{\tilde{H}_{0}^{1}}^{2} & =\int_{\alpha}^{\beta}\left|\frac{d}{d t}\left[\int_{\alpha}^{\beta} f_{x}\left(t, \tau, x_{0}(\tau)\right)\left(T^{k-1} w\right)(\tau) d \tau\right]\right|^{2} d t \\
& =\int_{\alpha}^{\beta}\left|\int_{\alpha}^{\beta} f_{x t}\left(t, \tau, x_{0}(\tau)\right)\left(T^{k-1} w\right)(\tau) d \tau\right|^{2} d t
\end{aligned}
$$




$$
\begin{aligned}
& \leq \int_{\alpha}^{\beta}\left[(\beta-\alpha) l_{\rho}(\beta-\alpha)^{k-1} l_{\rho}^{k-1} M\right]^{2} d t \\
& =(\beta-\alpha)^{2 k+1} l_{\rho}^{2 k} M^{2} \leq(\beta-\alpha)^{2 k+2} l_{\rho}^{2 k}\|w\|_{\tilde{H}_{0}^{1}}^{2},
\end{aligned}
$$

where $M=\|w\|_{\infty}$ and, hence, by an arbitrary choice of $w \in \widetilde{H}_{0}^{1}$, one gets

$$
\left\|T^{k}\right\|^{1 / k} \leq(\beta-\alpha)^{1+1 / k} l_{\rho}
$$

and consequently,

$$
r(T)=\limsup _{k \rightarrow \infty}\left\|T^{k}\right\|^{1 / k} \leq l_{\rho}(\beta-\alpha) .
$$

Theorem 4.2 leads to the inclusion $\sigma(T) \subset \bar{B}_{r(T)}$ with $r(T)$ defined in (4.6) and then, for all $\lambda \in \mathbb{R}$ such that $|\lambda|>r(T)$, we have $\lambda \in \rho(T)$. Therefore, one can conclude that, for all $\lambda \in \mathbb{R}$ and $|\lambda|>l_{\rho}(\beta-\alpha)$, the operator $T-\lambda I$ is bijective on $\widetilde{H}_{0}^{1}$. To sum up, for any real $\lambda$ such that $|\lambda|>l_{\rho}(\beta-\alpha)$, any $x_{0} \in \widetilde{H}_{0}^{1}$ such that $\left\|x_{0}\right\|_{\infty} \leq \rho$ and any $w \in \widetilde{H}_{0}^{1}$, there exists a unique function $h \in \widetilde{H}_{0}^{1}$ such that

$$
(T+\lambda I) h=w
$$

that is our linear equation,

$$
\lambda h(t)+\int_{\alpha}^{\beta} f_{x}\left(t, \tau, x_{0}(\tau)\right) h(\tau) d \tau=w(t),
$$

which ends the proof.

Remark 4.5. It should be emphasized that global assumption $A 4$ can be equivalently expressed as $|\lambda|>l_{\infty}(\beta-\alpha)$ where $l_{\infty}=$ $\sup _{(t, \tau) \in P, x \in \mathbb{R}^{n}}\left|f_{x t}(t, \tau, x)\right|$. This is a stronger assumption than the local solvability assumption from Lemma 4.4. This stronger assumption is, however, required for the solvability of the original nonlinear integral Urysohn equation, i.e., $F(x)=z$. In fact, for the solvability of the original equation, the choice of $\lambda$ must be independent of $\rho, l_{\rho}$ and $x_{0}$, i.e., 'local variables' existing only in the proof of Lemma 4.4 on the solvability of the linearized integral equation but not in the statement of the main theorem for the nonlinear Urysohn integral equation; see, Theorem 6.1 and Corollary 6.2. 
Remark 4.6. One can think of the rescaled operator $F^{\prime}\left(x_{0}\right) / \lambda$ at any point $x_{0}$ for $F$ defined by (1.3) as the perturbation of the identity. If we rescale the equation (1.1) by the $\lambda$ factor

$$
0=(z-F(x)) / \lambda,
$$

then, indeed, the operator $I-F^{\prime}\left(x_{0}\right) / \lambda$ has a norm smaller than one, since, due to assumptions $A 1(c), A 2(c)$ and $A 3$, we have (4.4) which, combined with the inequality from Lemma 4.4, guarantees the claim. Thus, the operator $F / \lambda$ is at any point some perturbation of the identity by the contraction and, therefore, a homeomorphism. But it is worth underlining that our main goal is to establish $F$ to be a global diffeomorphism not a local homeomorphism. In fact, the local, but not global, existence, uniqueness and continuous dependence of solutions to our equation follows from the direct application of the Banach fixed point theorem to the operator $(-F+z) / \lambda+l$ for any fixed $z$. Indeed, this operator maps any ball $B_{\rho}\left(x_{0}\right)$ into itself provided that $\rho$ is sufficiently large $\left(\rho>\left(\left\|F\left(x_{0}\right)-\lambda x_{0}-z\right\|\right) /((1-L) \lambda)\right)$ and, additionally, satisfies the contraction condition with the constant $L=(\beta-\lambda) l_{\rho} /|\lambda|<1$, as was noted before. The contraction constant $L$ depends on $\rho$.

\section{The Palais-Smale condition for the functional related to} the Urysohn operator. In this section, we demonstrate that assumption (b) of Theorem 2.1 is valid. Let us consider as in Theorem 2.1 the functional $\Psi_{y}: \widetilde{H}_{0}^{1} \rightarrow \mathbb{R}$ of the form

$$
\begin{aligned}
\Psi_{y}(x) & =\frac{1}{2}\|F x-y\|_{\tilde{H}_{0}^{1}}^{2} \\
& =\frac{1}{2} \int_{\alpha}^{\beta}\left|\lambda x^{\prime}(t)+\int_{\alpha}^{\beta} f_{t}(t, \tau, x(\tau)) d \tau-y^{\prime}(t)\right|^{2} d t
\end{aligned}
$$

where $y \in \widetilde{H}_{0}^{1}$ is an arbitrary function. Assumptions imposed on function $f$ guarantee that the functional (5.1) satisfies the Palais-Smale condition. Indeed, we have the following lemma.

Lemma 5.1. Under assumptions $A 1, A 2, A 3$ and $A 5$ on $f$, for any $y \in \widetilde{H}_{0}^{1}$, the functional $\Psi_{y}$ satisfies the Palais-Smale condition. 
Proof. We first demonstrate that, for any $y \in \widetilde{H}_{0}^{1}$, the functional $\Psi_{y}$ is coercive, i.e., for any $y \in \widetilde{H}_{0}^{1}, \Psi_{y}(x) \rightarrow \infty$ provided that $\|x\|_{\tilde{H}_{0}^{1}} \rightarrow \infty$. Since the functional $\Psi_{y}$ is coercive for any $y \in \widetilde{H}_{0}^{1}$ if and only if the functional $\Psi_{y}$ is coercive for $y=0$, we first show that the functional $\Psi_{0}$ is bounded from below. By the Schwarz inequality and the assumptions of this lemma, we get

$$
\begin{aligned}
\Psi_{0}(x)= & \frac{1}{2} \int_{\alpha}^{\beta}\left|\lambda x^{\prime}(t)+\int_{\alpha}^{\beta} f_{t}(t, \tau, x(\tau)) d \tau\right|^{2} d t \\
\geq & \frac{\lambda^{2}-\sqrt{2}(\beta-\alpha)|\lambda|\|a\|_{L^{2}}}{2}\|x\|_{\tilde{H}_{0}^{1}}^{2} \\
& -\sqrt{\beta-\alpha}|\lambda|\|b\|_{L^{2}}\|x\|_{\tilde{H}_{0}^{1}} .
\end{aligned}
$$

From $A 5(b)$ and the above estimate it follows that $\Psi_{0}(x) \rightarrow \infty$ if $\|x\|_{\tilde{H}_{0}^{1}} \rightarrow \infty$. Consequently, for any $y \in \widetilde{H}_{0}^{1}$, we have that $\Psi_{y}(x) \rightarrow \infty$ as $\|x\|_{\tilde{H}_{0}^{1}} \rightarrow \infty$. Now, fix $y \in \widetilde{H}_{0}^{1}$. It easy to see that the functional $\Psi_{y}$ has the form

$$
\begin{aligned}
\Psi_{y}(x)= & \frac{1}{2} \int_{\alpha}^{\beta}\left(\lambda^{2}\left|x^{\prime}(t)\right|^{2}+2\left\langle\lambda x^{\prime}(t), \int_{\alpha}^{\beta} f_{t}(t, \tau, x(\tau)) d \tau\right\rangle\right. \\
& +\left|\int_{\alpha}^{\beta} f_{t}(t, \tau, x(\tau)) d \tau\right|^{2}-2\left\langle\lambda x^{\prime}(t), y^{\prime}(t)\right\rangle \\
& \left.-2\left\langle\int_{\alpha}^{\beta} f_{t}(t, \tau, x(\tau)) d \tau, y^{\prime}(t)\right\rangle+\left|y^{\prime}(t)\right|^{2}\right) d t .
\end{aligned}
$$

Moreover, the functional $\Psi_{y}$ being a superposition of two $C^{1}$ class mappings is also of the same $C^{1}$ regularity class and its differential $\Psi_{y}^{\prime}(x)$ at $x \in \widetilde{H}_{0}^{1}$ is given, for $h \in \widetilde{H}_{0}^{1}$, by the formula

$$
\begin{aligned}
\Psi_{y}^{\prime}(x) h= & \int_{\alpha}^{\beta}\left[\lambda^{2}\left\langle x^{\prime}(t), h^{\prime}(t)\right\rangle+\left\langle\lambda h^{\prime}(t), \int_{\alpha}^{\beta} f_{t}(t, \tau, x(\tau)) d \tau\right\rangle\right. \\
& +\left\langle\lambda x^{\prime}(t), \int_{\alpha}^{\beta} f_{t x}(t, \tau, x(\tau)) h(\tau) d \tau\right\rangle \\
& +\left\langle\int_{\alpha}^{\beta} f_{t}(t, \tau, x(\tau)) d \tau, \int_{\alpha}^{\beta} f_{t x}(t, \tau, x(\tau)) h(\tau) d \tau\right\rangle
\end{aligned}
$$




$$
\left.-\left\langle\lambda h^{\prime}(t), y^{\prime}(t)\right\rangle-\left\langle\int_{\alpha}^{\beta} f_{t x}(t, \tau, x(\tau)) h(\tau) d \tau, y^{\prime}(t)\right\rangle\right] d t .
$$

Let $\left\{x_{k}\right\} \subset \widetilde{H}_{0}^{1}$ be a Palais-Smale sequence. We have proved that $\Psi_{y}$ is coercive, and hence the sequence $\left\{x_{k}\right\}$ is weakly compact as a bounded sequence in a reflexive space. Passing, if necessary, to a subsequence, one can assume that $x_{k} \rightarrow x_{0}$ weakly in $\widetilde{H}_{0}^{1}$ where $x_{0} \in \widetilde{H}_{0}^{1}$. Moreover, the weak convergence of the sequence $\left\{x_{k}\right\}$ in the space $\widetilde{H}_{0}^{1}$ implies the uniform convergence in $C$, i.e., $x_{k}(t) \rightrightarrows x_{0}(t)$ uniformly for $t \in[\alpha, \beta]$ as well as the weak convergence of its derivatives in $L^{2}$, i.e., $x_{k}^{\prime} \rightarrow x_{0}^{\prime}$ in $L^{2}$. Furthermore, a weakly convergent sequence $\left\{x_{k}^{\prime}\right\}$ in $L^{2}$ is bounded. It remains to prove that the sequence $\left\{x_{k}\right\}$ converges to $x_{0}$ in $\widetilde{H}_{0}^{1}$. By formula (5.2), a direct calculation leads to

$$
\left\langle\Psi_{y}^{\prime}\left(x_{k}\right)-\Psi_{y}^{\prime}\left(x_{0}\right), x_{k}-x_{0}\right\rangle=\lambda^{2}\left\|x_{k}-x_{0}\right\|_{\tilde{H}_{0}^{1}}^{2}+\sum_{i=1}^{6} G_{i}\left(x_{k}\right)
$$

where

$$
\begin{aligned}
G_{1}\left(x_{k}\right)= & \int_{\alpha}^{\beta}\left\langle\lambda\left(x_{k}^{\prime}(t)-x_{0}^{\prime}(t)\right),\right. \\
& \left.\int_{\alpha}^{\beta}\left[f_{t}\left(t, \tau, x_{k}(\tau)\right)-f_{t}\left(t, \tau, x_{0}(\tau)\right)\right] d \tau\right\rangle d t, \\
G_{2}\left(x_{k}\right)= & \int_{\alpha}^{\beta}\left\langle\lambda x_{k}^{\prime}(t),\right. \\
& \left.\int_{\alpha}^{\beta} f_{t x}\left(t, \tau, x_{k}(\tau)\right)\left(x_{k}(\tau)-x_{0}(\tau)\right) d \tau\right\rangle d t, \\
G_{3}\left(x_{k}\right)= & \int_{\alpha}^{\beta}\left\langle\int_{\alpha}^{\beta} f_{t}\left(t, \tau, x_{k}(\tau)\right) d \tau,\right. \\
& \left.\int_{\alpha}^{\beta} f_{t x}\left(t, \tau, x_{k}(\tau)\right)\left(x_{k}(\tau)-x_{0}(\tau)\right) d \tau\right\rangle d t, \\
G_{4}\left(x_{k}\right)= & -\int_{\alpha}^{\beta}\left\langle\int _ { \alpha } ^ { \beta } \left( f_{t x}\left(t, \tau, x_{k}(\tau)\right)\right.\right. \\
& \left.\left.-f_{t x}\left(t, \tau, x_{0}(\tau)\right)\right)\left(x_{k}(\tau)-x_{0}(\tau)\right) d \tau, y^{\prime}(t)\right\rangle d t,
\end{aligned}
$$




$$
\begin{aligned}
G_{5}\left(x_{k}\right)= & -\int_{\alpha}^{\beta}\left\langle\lambda x_{0}^{\prime}(t), \int_{\alpha}^{\beta} f_{t x}\left(t, \tau, x_{0}(\tau)\right)\left(x_{k}(\tau)-x_{0}(\tau)\right) d \tau\right\rangle d t \\
G_{6}\left(x_{k}\right)= & -\int_{\alpha}^{\beta}\left\langle\int_{\alpha}^{\beta} f_{t}\left(t, \tau, x_{0}(\tau)\right) d \tau\right. \\
& \left.\int_{\alpha}^{\beta} f_{t x}\left(t, \tau, x_{0}(\tau)\right)\left(x_{k}(\tau)-x_{0}(\tau)\right) d \tau\right\rangle d t
\end{aligned}
$$

Since $\Psi_{y}^{\prime}\left(z_{k}\right) \rightarrow 0$ and $x_{k} \rightarrow x_{0}$ weakly in $\widetilde{H}_{0}^{1}, \lim _{k \rightarrow \infty}\left\langle\Psi_{y}^{\prime}\left(x_{k}\right)-\right.$ $\left.\Psi_{y}^{\prime}\left(x_{0}\right), x_{k}-x_{0}\right\rangle=0$. We shall prove that, for $i=1,2, \ldots, 6$, $\lim _{k \rightarrow \infty} G_{i}\left(x_{k}\right)=0$. By the Schwarz inequality, we obtain

$$
\begin{aligned}
\left|G_{1}\left(x_{k}\right)\right|^{2} \leq & \lambda^{2} \int_{\alpha}^{\beta}\left|x_{k}^{\prime}(t)-x_{0}^{\prime}(t)\right|^{2} d t \\
& \times \int_{\alpha}^{\beta}\left[\int_{\alpha}^{\beta}\left|f_{t}\left(t, \tau, x_{k}(\tau)\right)-f_{t}\left(t, \tau, x_{0}(\tau)\right)\right| d \tau\right]^{2} d t .
\end{aligned}
$$

In the above inequality, the first integral is bounded, whereas the second one, by the Lebesgue theorem, is convergent to zero as $k \rightarrow \infty$, and therefore $G_{1}\left(x_{k}\right) \rightarrow 0$. Similar reasoning applies to other terms, i.e., by the Schwarz inequality, the uniform convergence of $\left\{x_{k}\right\}$ to $x_{0}$ in $C$ and the boundedness of $\left\{x_{k}^{\prime}\right\}$ in $L^{2}$, we can prove that $G_{i}\left(x_{k}\right) \rightarrow 0$ as $k \rightarrow \infty$ for $i=2,3,4,5,6$. Consequently, from (5.3), it follows that $x_{k} \rightarrow x_{0}$ in $\widetilde{H}_{0}^{1}$, which completes the proof.

\section{Main results for nonlinear Urysohn integral equations.} Applying Lemma 4.4 from Section 4, Lemma 5.1 from Section 5 and Theorem 2.1 presented in Section 2 we prove the existence, uniqueness and differentiable dependence on data results involving the following nonlinear Urysohn integral equations of the form:

$$
\lambda x(t)+\int_{\alpha}^{\beta} f(t, \tau, x(\tau)) d \tau=z(t),
$$

where $t \in[\alpha, \beta], \lambda \in \mathbb{R}, f: P \times \mathbb{R}^{n} \rightarrow \mathbb{R}^{n}, n \geq 1$ and $x, z \in \widetilde{H}_{0}^{1}$. The theorem to be proved is the following.

Theorem 6.1. If the function $f$ satisfies conditions $A 1-A 5$, then the 
operator $F: \widetilde{H}_{0}^{1} \rightarrow \widetilde{H}_{0}^{1}$ of the form

$$
F(x)(t)=\lambda x(t)+\int_{\alpha}^{\beta} f(t, \tau, x(\tau)) d \tau, \quad t \in[\alpha, \beta]
$$

is a diffeomorphism.

Proof. Setting $H=\widetilde{H}_{0}^{1}$ and $\mathcal{F}=F$, from Lemma 4.4 and Remark 4.5, we infer that the operator $F$ satisfies assumption (a) of Theorem 2.1. Lemma 5.1 assures us that, for any $y \in \widetilde{H}_{0}^{1}$, the functional $\Psi_{y}(x)=(1 / 2)\|F(x)-y\|_{\tilde{H}_{0}^{1}}^{2}$ satisfies the Palais-Smale condition and consequently assumption (b) of Theorem 2.1 is fulfilled. Therefore, the operator $F: \widetilde{H}_{0}^{1} \rightarrow \widetilde{H}_{0}^{1}$ defined by (6.2) is a diffeomorphism.

Theorem 6.1 can be formulated in the equivalent form focusing on solvability, uniqueness and continuous dependence issues for the related nonlinear Urysohn integral equations. Here is a more explicit statement of what the theorem asserts for this type of equation.

Corollary 6.2. If the function $f$ satisfies conditions $A 1-A 5$, then for any $z \in \widetilde{H}_{0}^{1}$ the nonlinear Urysohn integral system (6.1) possesses a unique solution $x=x_{z} \in \widetilde{H}_{0}^{1}$, and the operator $\widetilde{H}_{0}^{1} \ni z \rightarrow x_{z} \in \widetilde{H}_{0}^{1}$ is continuously Fréchet differentiable.

Let us now present the application of Theorem 6.1 to some specific nonlinear integral Urysohn operator and to the related nonlinear Urysohn integral equations.

Example 6.3. Let us consider the following operator

$$
F(x)(t)=\lambda x(t)+\int_{\alpha}^{\beta} A(t, \tau) \ln \left(1+B(t, \tau) x^{2}(\tau)\right) d \tau, \quad t \in[\alpha, \beta],
$$

with functions $A, B \in C^{1}(P, \mathbb{R})$ such that $A(\alpha, \tau)=0$ for $\tau \in[\alpha, \beta]$ and $B(t, \tau)>0$ on $P=[\alpha, \beta] \times[\alpha, \beta]$. Hence, the function $f: P \times \mathbb{R} \rightarrow \mathbb{R}$ has the form

$$
f(t, \tau, x)=A(t, \tau) \ln \left(1+B(t, \tau) x^{2}\right) .
$$


Let us notice that

$$
f_{t}(t, \tau, x)=A_{t}(t, \tau) \ln \left(1+B(t, \tau) x^{2}\right)+A(t, \tau) \frac{B_{t}(t, \tau) x^{2}}{1+B(t, \tau) x^{2}} .
$$

Since $\ln \left(1+z^{2}\right) \leq|z|$ and $1 /\left(1+w^{2}\right) \leq 1 / w^{2}$, we have

$$
\left|f_{t}(t, \tau, x)\right| \leq\left|A_{t}(t, \tau)\right| \sqrt{B(t, \tau)}|x|+\frac{|A(t, \tau)|\left|B_{t}(t, \tau)\right|}{B(t, \tau)} .
$$

Let us define

$$
a(t, \tau)=\left|A_{t}(t, \tau)\right| \sqrt{B(t, \tau)}
$$

and

$$
b(t, \tau)=\left(|A(t, \tau)|\left|B_{t}(t, \tau)\right|\right) /(B(t, \tau)) .
$$

Then $a, b \in L^{2}\left(P, \mathbb{R}^{+}\right)$, and condition $A 5(a)$ is fulfilled. Assuming

$$
\|a\|_{L^{2}\left(P, \mathbb{R}^{+}\right)}=\left\|\left|A_{t}(t, \tau)\right| \sqrt{B(t, \tau)}\right\|_{L^{2}\left(P, \mathbb{R}^{+}\right)}<\frac{\sqrt{2}|\lambda|}{2(\beta-\alpha)},
$$

we can guarantee that assumption $A 5(b)$ is also satisfied. Furthermore,

$$
\begin{gathered}
f_{x}(t, \tau, x)=A(t, \tau) \frac{2 B(t, \tau) x}{1+B(t, \tau) x^{2}} \\
f_{x t}(t, \tau, x)=A_{t}(t, \tau) \frac{2 B(t, \tau) x}{1+B(t, \tau) x^{2}}+A(t, \tau) B_{t}(t, \tau) \frac{2 x}{\left(1+B(t, \tau) x^{2}\right)^{2}}
\end{gathered}
$$

and

$$
\left|f_{x t}(t, \tau, x)\right| \leq\left|A_{t}(t, \tau)\right| \sqrt{B(t, \tau)}+|A(t, \tau)| \frac{\left|B_{t}(t, \tau)\right|}{B(t, \tau)} .
$$

Consequently, if we assume that

$$
\left|A_{t}(t, \tau)\right| \sqrt{B(t, \tau)}+|A(t, \tau)| \frac{\left|B_{t}(t, \tau)\right|}{B(t, \tau)}<\frac{|\lambda|}{\beta-\alpha},
$$

then condition $A 4$ holds. Thus, function $f$ satisfies assumptions $A 1-A 5$ and Theorem 6.1 implies that, for any $z \in \widetilde{H}_{0}^{1}$, the equation

$$
\lambda x(t)+\int_{\alpha}^{\beta} A(t, \tau) \ln \left(1+B(t, \tau) x^{2}(\tau)\right) d \tau=z(t), \quad t \in[\alpha, \beta]
$$


possesses a unique solution $x=x_{z} \in \widetilde{H}_{0}^{1}$ and the operator $\widetilde{H}_{0}^{1} \ni z \rightarrow$ $x_{z} \in \widetilde{H}_{0}^{1}$ is continuously Fréchet differentiable.

Remark 6.4. In Example 6.3, one can take

$$
A(t, \tau)=a_{0} t^{\gamma} \tau^{\eta}(t-\tau)^{\kappa}
$$

and

$$
B(t, \tau)=b_{0} t^{\nu} \tau^{\mu}(t-\tau)^{\chi}+b_{1}
$$

for suitably large positive $\gamma, \eta, \kappa, \nu, \mu, \chi$, sufficiently small positive $a_{0}$, $b_{0}, b_{1}$ and $[\alpha, \beta] \subset \mathbb{R}^{+}$.

7. Nonlinear Urysohn integral equations with controls. Now, let us focus our attention on a nonlinear control system of Urysohn type

$$
\lambda x(t)+\int_{\alpha}^{\beta} g(t, \tau, x(\tau), u(\tau)) d \tau=z(t),
$$

where $t \in[\alpha, \beta], \lambda \in \mathbb{R}, g: P \times \mathbb{R}^{n} \times \mathbb{R}^{m} \rightarrow \mathbb{R}^{n}, n \geq 1, m \geq 1, x, z \in \widetilde{H}_{0}^{1}$ and $u \in L^{2}$.

To obtain solvability, uniqueness and continuous dependence results for control system (7.1), it is enough to demonstrate that the operator

$$
G: \widetilde{H}_{0}^{1} \times L^{2} \times \widetilde{H}_{0}^{1} \longrightarrow \widetilde{H}_{0}^{1}
$$

of the form:

$$
G(x, u, z)(t)=\lambda x(t)+\int_{\alpha}^{\beta} g(t, \tau, x(\tau), u(\tau)) d \tau-z(t), \quad t \in[\alpha, \beta]
$$

satisfies the assumptions of Theorem 2.3 with $H=\widetilde{H}_{0}^{1}$ and $Y=$ $L^{2} \times \widetilde{H}_{0}^{1}$.

In that case, on the function $g$ we impose analogous conditions similar to those appearing in Section 3:

B1(a) the function $g(\cdot, \tau, \cdot, \cdot)$ is continuous on the set $Q^{\prime}:=[\alpha, \beta] \times$ $\mathbb{R}^{n} \times \mathbb{R}^{m}$ for almost every $\tau \in[\alpha, \beta]$,

B1(b) there exists $g_{t}(\cdot, \tau, \cdot, \cdot)$, and it is continuous on $Q^{\prime}$ for almost every $\tau \in[\alpha, \beta]$, 
B1(c) there exists $g_{x}(\cdot, \tau, \cdot, \cdot)$, and it is continuous on set $Q^{\prime}$ for almost every $\tau \in[\alpha, \beta]$,

B1(d) there exists $g_{t x}(\cdot, \tau, \cdot, \cdot)$, and it is continuous on set $Q^{\prime}$ for almost every $\tau \in[\alpha, \beta]$,

B1(e) there exists $g_{u}(\cdot, \tau, \cdot, \cdot)$, and it is continuous on set $Q^{\prime}$ for almost every $\tau \in[\alpha, \beta]$,

B2(a) the function $g(t, \tau, x, u)$ is measurable with respect to $\tau$ for $(t, x, u) \in Q^{\prime}$ and locally bounded with respect to $x$ for $(t, \tau) \in$ $P$ and $u \in \mathbb{R}^{m}$, i.e., for every $\rho>0$, there exists $l_{\rho}>0$ such that, for $(t, \tau) \in P$ and $x \in\left\{p \in \mathbb{R}^{n} ;|p| \leq \rho\right\}$ and $u \in\left\{p \in \mathbb{R}^{m} ;|p| \leq \rho\right\}$ we have $|g(t, \tau, x, u)| \leq l_{\rho}$,

B2(b) the function $g_{t}(t, \tau, x, u)$ satisfies B2(a) with $g_{t}$ instead of $g$,

B2(c) the function $g_{x}(t, \tau, x, u)$ satisfies B2(a) with $g_{x}$ instead of $g$,

B2(d) the function $g_{t x}(t, \tau, x, u)$ satisfies B2(a) with $g_{t x}$ instead of $g$;

B2(e) the function $g_{u}(t, \tau, x, u)$ satisfies B2(a) with $g_{u}$ instead of $g$;

B3 $g(\alpha, \tau, x, u)=0, g_{x}(\alpha, \tau, x, u)=0$ and $g_{u}(\alpha, \tau, x, u)=0$ for any $x \in \mathbb{R}^{n}, u \in \mathbb{R}^{m}$ and almost every $\tau \in[\alpha, \beta] ;$

B4 $\left|g_{x t}(t, \tau, x, u)\right|<|\lambda| /(\beta-\alpha)$ for any $x \in \mathbb{R}^{n}, u \in \mathbb{R}^{m}$ and $(t, \tau) \in P$

$\mathrm{B} 5(\mathrm{a})\left|g_{t}(t, \tau, x, u)\right| \leq a(t, \tau)|x|+b(t, \tau)$ where $(t, \tau) \in P, a, b \in$ $L^{2}(P, \mathbb{R})$, for any $x \in \mathbb{R}^{n}, u \in \mathbb{R}^{m}$ and $(t, \tau) \in P$;

B5(b) $\|a\|_{L^{2}(P, \mathbb{R})}<(\sqrt{2}|\lambda|) /(2(\beta-\alpha))$.

Under assumptions $B 1(a), B 1(b), B 1(c), B 2(a), B 2(b), B 2(c)$ and $B 3$ one can verify that $G$ is well defined and is of the $C^{1}$ class. Furthermore, for any $h \in \widetilde{H}_{0}^{1}$ and $(k, l) \in L^{2} \times \widetilde{H}_{0}^{1}$,

$$
\begin{aligned}
G_{x}(x, u, z) h(t) & =\lambda h(t)+\int_{\alpha}^{\beta} g_{x}(t, \tau, x(\tau), u(\tau)) h(\tau) d \tau \\
G_{(u, z)}(x, u, z)(k, l)(t) & =\int_{\alpha}^{\beta} g_{u}(t, \tau, x(\tau), u(\tau)) k(\tau) d \tau-l(t)
\end{aligned}
$$

are the differentials of $G$ with respect to $x$ and $(u, z)$, respectively.

Let us fix a function $(u, z) \in L^{2} \times \widetilde{H}_{0}^{1}$. It is easy to check that, if the function $g$ satisfies B1-B5, then the mapping $\widetilde{g}: P \times \mathbb{R}^{n} \rightarrow \mathbb{R}^{n}$ of the form

$$
\widetilde{g}(t, \tau, x)=g(t, \tau, x, u(\tau))
$$


meets conditions A1-A5. Since

$$
\begin{aligned}
\lambda h^{\prime}(t)+\int_{\alpha}^{\beta} g_{x}(t, \tau, x(\tau), u(\tau)) h(\tau) d \tau & \\
& =\lambda h^{\prime}(t)+\int_{\alpha}^{\beta} \tilde{g}_{x}(t, \tau, x(\tau)) h(\tau) d \tau
\end{aligned}
$$

for $t \in[\alpha, \beta]$; therefore, just as in Lemma 4.4, one can demonstrate that the mapping $G_{x}(x, u, z)$ is bijective for any $x \in \widetilde{H}_{0}^{1}$. Furthermore, similarly as in the proof of Lemma 5.1, one can deduce that, for any $(u, z) \in L^{2} \times \widetilde{H}_{0}^{1}$, the functional

$$
\begin{aligned}
\Psi_{(u, z)}(x) & =\frac{1}{2}\|G(x, u, z)\|_{\tilde{H}_{0}^{1}}^{2} \\
& =\frac{1}{2} \int_{\alpha}^{\beta}\left|\lambda x^{\prime}(t)+\int_{\alpha}^{\beta} g_{t}(t, \tau, x(\tau), u(\tau)) d \tau-z^{\prime}(t)\right|^{2} d t
\end{aligned}
$$

satisfies the Palais-Smale condition. Thus, for the operator $G(x, y)$ in (7.2) with $y=(u, z)$, it is enough to apply Theorem 2.3 in the spaces $H=\widetilde{H}_{0}^{1}$ and $Y=L^{2} \times \widetilde{H}_{0}^{1}$ to obtain the following theorem.

Theorem 7.1. If the function g satisfies B1-B5, then the nonlinear Urysohn integral equations with control (7.1) possesses a unique solution $x=x_{(u, z)} \in \widetilde{H}_{0}^{1}$, and the operator $L^{2} \times \widetilde{H}_{0}^{1} \ni(u, z) \rightarrow x_{(u, z)} \in \widetilde{H}_{0}^{1}$ is continuously Fréchet differentiable on $L^{2} \times \widetilde{H}_{0}^{1}$.

Let us give the following application of the above theorem.

Example 7.2. Let us consider the following equation:

$$
\lambda x(t)+\int_{0}^{1}\left(\frac{1}{18} t^{6} \sqrt{\tau} \sin x(\tau)+\frac{2}{3} \sqrt{t^{3}} \tau^{3} \sin ^{2} x(\tau) \sin u(\tau)\right) d \tau=z(t)
$$

for $t \in[0,1]$ where $|\lambda|>4 / 3$ and the function $g: P \times \mathbb{R} \times \mathbb{R} \rightarrow \mathbb{R}$ with $P=[0,1] \times[0,1]$ has the form

$$
g(t, \tau, x, u)=\frac{1}{18} t^{6} \sqrt{\tau} \sin x+\frac{2}{3} \sqrt{t^{3}} \tau^{3} \sin ^{2} x \sin u,
$$


and moreover,

$$
\begin{aligned}
& g_{x}(t, \tau, x, u)=\frac{1}{18} t^{6} \sqrt{\tau} \cos x+\frac{2}{3} \sqrt{t^{3}} \tau^{3} \sin 2 x \sin u \\
& g_{u}(t, \tau, x, u)=\frac{2}{3} \sqrt{t^{3}} \tau^{3} \sin ^{2} x \cos u,
\end{aligned}
$$

that is, the function $g$ satisfies B1-B3. Furthermore,

$$
\begin{aligned}
\left|g_{x t}(t, \tau, x, u)\right| & =\left|\frac{1}{3} t^{5} \sqrt{\tau} \cos x+\sqrt{t} \tau^{3} \sin 2 x \sin u\right| \\
& \leq \sqrt{t} \tau^{3}\left(\frac{1}{3} t^{2}+1\right)<|\lambda|
\end{aligned}
$$

thus, condition B4 holds. Because of the estimate

$$
\begin{aligned}
\left|g_{t}(t, \tau, x, u)\right| & =\left|\frac{1}{3} t^{5} \sqrt{\tau} \sin x+\sqrt{t} \tau^{3} \sin ^{2} x \sin u\right| \\
& \leq\left|\frac{1}{3} t^{5} \sqrt{\tau} \sin x\right|+\left|\sqrt{t} \tau^{3} \sin ^{2} x \sin u\right| \\
& \leq \sqrt{t} \tau^{3}\left(\frac{1}{3} t^{2}+1\right),
\end{aligned}
$$

it is easy to observe that condition B5 is satisfied as $|\lambda|>4 / 3$. Thus, from Theorem 2.3, it follows that, for any $(u, z) \in L^{2} \times \widetilde{H}_{0}^{1}$, there exists a unique solution $x_{(u, z)}$ of the equation (7.2) and the mapping $\Lambda: L^{2} \times \widetilde{H}_{0}^{1} \ni(u, z) \rightarrow x_{(u, z)} \in \widetilde{H}_{0}^{1}$ is of the $C^{1}$ class with the differential $\Lambda^{\prime}(u, z)$ at a point $(u, z) \in L^{2} \times \widetilde{H}_{0}^{1}$ of the form

$$
L^{2} \times \widetilde{H}_{0}^{1} \ni(k, l) \longrightarrow w_{(k, l)} \in \widetilde{H}_{0}^{1}
$$

where $w_{(k, l)}$ is such that

$$
\begin{aligned}
& \lambda w_{(k, l)}(t) \\
& +\int_{0}^{1}\left(\frac{1}{18} t^{6} \sqrt{\tau} \cos x_{(u, z)}(\tau)\right. \\
& \left.+\frac{2}{3} \sqrt{t^{3}} \tau^{3} \sin 2 x_{(u, z)}(\tau) \sin u(\tau)\right) w_{(k, l)}(\tau) d \tau \\
& =-\int_{0}^{1} \frac{2}{3} \sqrt{t^{3}} \tau^{3} \sin ^{2} x_{(u, z)}(\tau) \cos u(\tau) k(\tau) d \tau+l(t)
\end{aligned}
$$


for $t \in[0,1]$.

8. Concluding remarks. We have obtained existence and uniqueness results for the system of the nonlinear Urysohn equations as well as differentiable dependence of solutions on parameters, see Theorem 6.1, Corollary 6.2 and Theorem 7.1. Thus, in other words, our problem is well posed and robust, cf., [20]. The main tool in the proof of the main result of the paper is the application of the mountain pass theorem presented in Section 2.

Integral operators and integral equations are most commonly examined in the space of square-integrable functions. Under suitable conditions, one usually proves the existence and uniqueness theorems. However, in this paper, the integral Urysohn operator $F$ is defined on the space $\widetilde{H}_{0}^{1}$. In the proof of Lemma 5.1 we have used the compactness of the embedding of the space $\widetilde{H}_{0}^{1}$ into the space $C$. This compact embedding implies that any weakly convergent sequence in $\widetilde{H}_{0}^{1}$ is uniformly convergent in $C$ in the sup-norm. Apparently, in the case of the $L^{2}$ space such an implication does not hold. Therefore, one cannot prove, at least with the method applied herein, that the operator $F: L^{2} \rightarrow L^{2}$ is a diffeomorphism but one could argue that it is a homeomorphism.

In Sections 3-6, we have shown, step by step, that assumptions A1A5 imply some sufficient conditions for the operator $F: \widetilde{H}_{0}^{1} \rightarrow \widetilde{H}_{0}^{1}$ defined by (1.3) to be a diffeomorphism, as stated in Theorem 6.1. From Corollary 6.2, the equation $F(x)=z$ possesses a unique solution that depends continuously differentiable on parameter $z$. Moreover, we have demonstrated in Section 7 that conditions B1-B5 imply that the assumptions of the global implicit function theorem involving the nonlinear Urysohn integral operator $G$ defined in (1.4) are satisfied, and therefore the equation $G(x, u, z)=0$ possesses a unique solution that depends in a continuous differentiable manner on parameters $(u, z)$.

Differences between the results for Urysohn and Hammerstein equations considered in [2] should be underlined. It should be emphasized that they are incomparable. In the Hammerstein case [2], the function $f$ has a more specific form with separated variables. However, the results obtained for the Hammerstein equations allow the singular kernels and thus do not follow from the results presented herein. The approach, 
however, is pretty similar. The results for equations involving Volterra operators also being the special case of the Urysohn operators can be deduced from this paper, so this is an extension of the results from [3].

It would also be interesting to consider, instead of investigating the linear operator $F^{\prime}\left(x_{0}\right)$ directly, the nonlinear operator $F$ by means of nonlinear spectral theory, where $F$ is defined by (1.3). For example, one could try to apply the Kachurovskij spectrum, cf., [15], for Lipschitz continuous operators, or the Neuburger spectrum, cf., [16], for $C^{1}$ operators. This might be an interesting direction for a subsequent paper.

Acknowledgments. The author would like to thank the anonymous referees for valuable remarks which improved the quality of the paper.

\section{REFERENCES}

1. A. Ambrosetti and P.H. Rabinowitz, Dual variational methods in critical point theory and applications, J. Funct. Anal. 14 (1973), 349-381.

2. D. Bors, Global solvability of Hammerstein equations with applications to BVP involving fractional Laplacian, Abstr. Appl. Anal, 240863 (2013), 10 pages.

3. D. Bors, A. Skowron and S. Walczak, Systems described by Volterra type integral operators, Discr. Cont. Dynam. Syst. 19 (2014), 2401-2416.

4. H. Brezis and F.E. Browder, Existence theorems for nonlinear integral equations of Hammerstein type, Bull. Amer. Math. Soc. 81 (1975), 73-78.

5. , Nonlinear integral equations and systems of Hammerstein type, Adv. Math. 18 (1975), 115-147.

6. C. Corduneanu, Integral equations and applications, Cambridge University Press, Cambridge, 1991.

7. C.E. Chidume and Y. Shehu, Strong convergence theorem for approximation of solutions of equations of Hammerstein type, Nonlin. Anal. 75 (2012), 5664-5671.

8. C.E. Chidume and H. Zegeye, Approximation of solutions of nonlinear equations of Hammerstein type in Hilbert space, Proc. Amer. Math. Soc. 133 (2004), 851-858.

9. D.G. deFigueiredo and C.P. Gupta, On the variational method for the existence of solutions of nonlinear equations of Hammerstein type, Proc. Amer. Math. Soc. 40 (1973), 470-476.

10. M.A. El-Ameen and M. El-Kady, A new direct method for solving nonlinear Volterra-Fredholm-Hammerstein integral equations via optimal control problem, J. Appl. Math. (2012), Article ID 714973, doi:10.1155/2012/714973.

11. G. Gripenberg, S.-O. Londen and O. Staffans, Volterra integral and functional equations, Cambridge University Press, Cambridge, 1990. 
12. D. Idczak, A global implicit function theorem and its applications to functional equations, Discr. Cont. Dynam. Syst. 19 (2014), 2549-2556.

13. D. Idczak, A. Skowron and S. Walczak, On the diffeomorphisms between Banach and Hilbert spaces, Adv. Nonlin. Stud. 12 (2012), 89-100.

14. A.D. Ioffe and V.M. Tihomirov, Theory of extremal problems, NorthHolland, Amsterdam, 1979.

15. R.I. Kachurovskij, Regular points, spectrum and eigenfunctions of nonlinear operators, Dokl. Akad. Nauk 188 (1969), 274-277 (in Russian); Sov. Math. Dokl. 100 (1969), 1101-1105 (in English).

16. J.W. Neuburger, Existence of a spectrum for nonlinear transformations, Pac. J. Math. 31 (1969), 157-159.

17. D. Pascali and S. Sburlan, Nonlinear mappings of monotone type, Editura Academiae, Bucharest, Romania, 1978.

18. P.H. Rabinowitz, Minimax methods in critical point theory with applications to differential equations, American Mathematical Society, Providence, 1986.

19. M. Reed and B. Simon, Methods of modern mathematical physics, Academic Press, New York, 1980.

20. R.S. Sánchez-Peńa and M. Sznaier, Robust systems theory and applications, Wiley-Interscience, New York, 1998.

21. R. Stańczy, Hammerstein equations with an integral over a noncompact domain, Ann. Polon. Math. 69 (1998), 49-60.

22. M. Willem, Minimax theorems, Birkhauser, Boston, 1996.

23. K. Yosida, Functional analysis, Springer-Verlag, Berlin, 1965.

Faculty of Mathematics and Computer Science, University of Lodz, Banacha 22, 90-238 Lodz, Poland

Email address: bors@math.uni.lodz.pl 